Fernanda Carolina Pereira, O Efeito dos Exergames na Motivação para a Prática de Atividade Física... Adriane Beatriz de S. Serapião e

José Silvio Govone

\title{
O EFEITO DOS EXERGAMES NA MOTIVAÇÃO PARA A PRÁTICA DE ATIVIDADE FÍSICA EM JOVENS UNIVERSITÁRIOS
}

\author{
Recebido em: $14 / 08 / 2016$
}

Aceito em: 31/05/2017

Fernanda Carolina Pereira

Adriane Beatriz de Souza Serapião

José Silvio Govone

Universidade Estadual Paulista "Julio de Mesquita Filho"

Rio Claro - SP - Brasil

RESUMO: Os exergames (EXGs), jogos virtuais que englobam o movimento humano em sua prática, têm funcionado atualmente como um intermédio entre a vida extremamente tecnológica e a inatividade física. Os objetivos do presente estudo foram identificar os principais motivos pelos quais as pessoas jogam videogames e EXGs, correlacionando-os aos motivos pelos quais elas praticam atividades físicas e esportes. Participaram do estudo 18 voluntários de ambos os gêneros, com idade média de 22,7 \pm 2,4 anos. Foi utilizado o Inventário de Motivação, Jogos Virtuais e Prática de Atividades Físicas/Esportes. Os resultados proporcionaram estabelecer conexões entre a influência das novas tecnologias, com enfoque específico nos jogos virtuais e EXGs, os estados emocionais, mais especificamente a motivação e a prática de exercícios e atividades físicas.

PALAVRAS CHAVE: Atividades de Lazer. Motivação. Educação Física e Treinamento.

\section{THE EFFECT OF EXERGAMES IN MOTIVATION FOR PHYSICAL ACTIVITY IN UNIVERSITY STUDENTS}

ABSTRACT: The exergames (EXGs), virtual games that involve the human movement in their practice, have now acted as an intermediary between highly technological life and physical inactivity. The objectives of this study were to identify the main reasons why people play video games and EXGs, correlating them to why they practice physical activities and sports. Study participants were 18 volunteers of both genders, with a mean age of $22.7 \pm 2.4$ years. Inventory of Motivation, Virtual Games and Practice of Physical Activities/Sports was used. The results provide connections between the influence of new technologies, with a specific focus on virtual and EXGs games, emotional states, specifically the motivation and the practice of physical exercises and activities.

KEYWORDS: Leisure Activities. Motivation. Physical Education and Training. 
Fernanda Carolina Pereira, O Efeito dos Exergames na Motivação para a Prática de Atividade Física... Adriane Beatriz de S. Serapião e

José Silvio Govone

\section{Introdução}

A tecnologia tem avançado em grande velocidade nas últimas décadas. Este avanço permitiu com que as pessoas levassem uma vida com maior conforto, porém com poucas horas dedicadas à prática de atividades físicas e lazer. Este quadro, quando associado ao estresse da vida cotidiana e aos hábitos alimentares inadequados, provoca ameaças à saúde, ao bem estar e à qualidade de vida das pessoas.

A literatura relata que crianças passam de 2,45 a 3,30 horas/dia, ou seja, uma média de 19,39 a 24,45 horas/semana em frente à televisão (WANG e PERRY, 2006), e destas, $74 \%$, entre 11 e 16 anos, jogam videogame de três a sete vezes por semana, por um período de no mínimo 2 horas/sessão (MADDISON et al., 2009).

Este longo período de tempo em que crianças e jovens se utilizam das mídias digitais de forma passiva, se utilizado de forma ativa, poderia aumentar o gasto energético e também auxiliar no prazer e divertimento das mesmas.

Neste contexto, surgem os exergames (EXGs), jogos virtuais que englobam o movimento humano em sua prática, servindo assim, como o intermédio entre a vida extremamente tecnológica e a inatividade física (TROUT e CHRISTIE, 2007). Quando a experiência de se envolver com EXGs são vista como algo agradável e divertido (ao invés de ser vista como um treino ou um exercício sistematizado), isto surge como um fator benéfico àquelas pessoas que normalmente têm um estilo de vida sedentário, proporcionando uma alternativa em direção a um estilo de vida fisicamente mais ativo.

É muito importante evidenciar que, talvez, esteja aí um possível caminho para a Educação Física recuperar o interesse dos alunos que não gostam de esportes ou de pessoas que não participam de atividades físicas regularmente, como sugere Ennis (2013) em seu trabalho, que analisa as implicações da utilização dos EXGs nos 
Fernanda Carolina Pereira, O Efeito dos Exergames na Motivação para a Prática de Atividade Física... Adriane Beatriz de S. Serapião e

José Silvio Govone

currículos escolares no século XXI. Em um estudo anterior, Ennis (2010) sugere que os EXGs funcionam como atrativo para aqueles alunos que se mostram relutantes em participar das aulas de Educação Física na escola. Segundo a autora, atrair estes alunos para a prática de atividades físicas é o primeiro passo para encorajá-los a participar de atividade física extraclasse voluntariamente e sem a insistência de um adulto.

Mas para que essa mudança de estilo de vida aconteça, é necessário que uma

mudança de pensamento ocorra. É neste sentido que o presente estudo buscou apoiar seus objetivos, que são identificar os principais motivos pelos quais as pessoas jogam videogames e EXGs, correlacionando-os aos motivos pelos quais elas praticam atividades físicas e esportes, buscando entender se as práticas dos EXGs causam alguma mudança na perspectiva de uma vida mais ativa dos voluntários participantes.

\section{Métodos}

O presente estudo foi aprovado no Comitê de Ética em Pesquisa local. Os voluntários que aceitaram participar do estudo assinaram termo de Consentimento Livre e Esclarecido. Participaram do estudo 18 voluntários, com idade média de $22,7 \pm 2,4$ anos, sendo quatro homens e 14 mulheres, sem antecedentes de doenças musculoesqueléticas, saudáveis e possuíam antropometria semelhante.

Foi aplicado o Questionário Internacional de Atividade Física (International Physical Activity Questionnaire - IPAQ - versão curta), cujas validações e reprodutibilidade foram descritas por Craig et al. (2003), e a partir dos resultados obtidos neste questionário, os voluntários foram alocados nos grupos sedentários, moderados e fisicamente ativos. Todos os voluntários foram submetidos a um período de intervenção de quatro semanas. 
Fernanda Carolina Pereira, O Efeito dos Exergames na Motivação para a Prática de Atividade Física... Adriane Beatriz de S. Serapião e

José Silvio Govone

Os procedimentos de intervenção foram realizados no período de quatro semanas, sendo a prática dos EXGs realizada duas vezes por semana. Cada sessão de intervenção teve a duração de 40 minutos, com períodos curtos de intervalo (1 a 5 minutos), tanto para descanso do participante, como para a mudança de jogos utilizados.

Foi aplicado um Inventário de Motivação, Jogos Virtuais e Prática de Atividades Físicas/Esportes, composto de 108 itens, dispostos em dois blocos de 54 itens, sendo um bloco referente à Motivação e Jogos Virtuais e outro bloco referente à Motivação para a Prática de Atividades Físicas e/ou Esportes. Os participantes deveriam responder aos itens dentro de uma escala de cinco pontos $(0=$ Discordo, $1=$ Concordo pouco, $2=$ Concordo, 3 = Concordo muito e 4 = Concordo totalmente). Os 54 itens de cada bloco do inventário compõem sete dimensões: (1) saúde e bem estar, (2) competitividade e desafios, (3) lazer e entretenimento, (4) sociabilidade, (5) competências e habilidades, (6) estados emocionais e (7) relaxamento. Em cada dimensão, obtém-se um escore que pode variar de 0 a 16. A aplicação deste inventário aconteceu em dois momentos, sendo o primeiro no primeiro dia de intervenção com EXGs (Pré) e o segundo no último dia de intervenção (Pós).

Para os procedimentos de intervenção realizados pelos grupos foi necessária a utilização de um console de videogame Xbox $360^{\circledR}$ acompanhado de um Kinect ${ }^{\text {TM}}$, um aparelho de televisão Sony LED 32 e o software do jogo escolhido para a prática em ambiente virtual. Foram utilizados os seguintes jogos para Xbox $360^{\circledR}$ : Kinect Sports, Kinect Sports Season 2, Motion Sports Adrenaline, Dance Central 3, Just Dance 2015, Wipeout 3, Hole in the Wall, Michael Phelps - Push The Limit, Your Shape Fitness Evolved 2012, Kinect Adventures. 
Fernanda Carolina Pereira, O Efeito dos Exergames na Motivação para a Prática de Atividade Física... Adriane Beatriz de S. Serapião e

José Silvio Govone

As coletas de dados foram realizadas em uma sala, localizada nas dependências do Departamento de Estatística, Matemática Aplicada e Computação (DEMAC - Unesp Campus de Rio Claro). A sala possui área de aproximadamente $11 \mathrm{~m}^{2}$, e dispõe de espaço suficiente para os voluntários jogarem os jogos no ambiente virtual do Kinect $^{\mathrm{TM}}$.

Os dados provenientes das entrevistas com o questionário IPAQ foram analisados de maneira descritiva, qualitativa e categorizada. Os dados foram processados utilizando um teste de Friedman para cada grupo (intragrupo) e KruskalWallis entre os grupos da amostra (intergrupo), para identificar se existiram efeitos significativos entre os fatores comparando os resultados no início e fim do treinamento com os EXGs. O nível de significância foi estabelecido em 5\%.

\section{Resultados}

A aplicação do IPAQ versão curta possibilitou a classificação dos 18 participantes em três grupos: seis indivíduos sedentários, seis moderados e seis fisicamente ativos. De acordo com uma entrevista, os participantes foram também alocados em dois outros grupos: nove jogadores de videogame (para aqueles que jogam acima de uma vez ao mês) e nove não jogadores de videogame (para aqueles que jogam uma vez ao mês ou menos).

As Tabelas 1 e 2 mostram os resultados estatísticos dos testes de Friedman na Tabela 1 e Kruskal-Wallis na Tabela 2 para todos os participantes, considerando as sete dimensões do Inventário de Motivação, Jogos Virtuais e Prática de Atividades Físicas/Esportes, e a Figura 1 mostra o gráfico representativo dos escores pré e pósintervenção com EXGs, em cada dimensão do mesmo Inventário. Nota-se que houve diferença significativa entre as respostas pré e pós para a dimensão Competências e 
Fernanda Carolina Pereira, O Efeito dos Exergames na Motivação para a Prática de Atividade Física... Adriane Beatriz de S. Serapião e

José Silvio Govone

Habilidades $(\mathrm{CH})(p=0,0001)$. Já o teste de variância de Kruskal-Wallis na Tabela 2 não resultou em diferenças significativas, o que indica que os participantes não alteraram suas opiniões acerca das temáticas Videogame e Atividades Físicas com a intervenção com EXGs.

Tabela 1: Teste de variância de Friedman nas condições pré e pós intervenção com EXGs, para todos os participantes, considerando as sete dimensões do Inventário de Motivação, Jogos Virtuais e Prática de Atividades Físicas/Esportes, $(* p<0,05)$.

\begin{tabular}{ll}
\hline Friedman & $(p)$ \\
\hline Lazer e Entretenimento (LE) & 0,8676 \\
\hline Saúde (SA) & 0,6171 \\
\hline Competências e Habilidades (CH) & $0,0001^{*}$ \\
\hline Sociabilidade (SC) & $\mathrm{ns}$ \\
\hline Competitividade e Desafios (CD) & 0,1824 \\
\hline Estados Emocionais (EE) & 0,6171 \\
\hline Relaxamento (RX) & 0,8676 \\
\hline
\end{tabular}

Tabela 2: Teste de variância de Kruskal-Wallis para todos os participantes, considerando as sete dimensões do Inventário de Motivação, Jogos Virtuais e Prática de Atividades Físicas/Esportes, $(* p<0,05)$.

\begin{tabular}{ll}
\hline Kruskal-Wallis & $(\boldsymbol{p})$ \\
\hline Lazer e Entretenimento (LE) & 0,9421 \\
\hline Saúde (SA) & 0,9345 \\
\hline Competências e Habilidades (CH) & 0,7938 \\
\hline Sociabilidade (SC) & 0,6112 \\
\hline Competitividade e Desafios (CD) & 0,4662 \\
\hline Estados Emocionais (EE) & 0,9606 \\
\hline Relaxamento (RX) & 0,8236 \\
\hline
\end{tabular}


Fernanda Carolina Pereira, O Efeito dos Exergames na Motivação para a Prática de Atividade Física... Adriane Beatriz de S. Serapião e

José Silvio Govone

Figura 1: Gráfico representativo dos escores pré e pós intervenção com EXGs, em cada dimensão do Inventário de Motivação, Jogos Virtuais e Prática de Atividades

Físicas/Esportes, para todos os participantes $(* p<0,05)$.

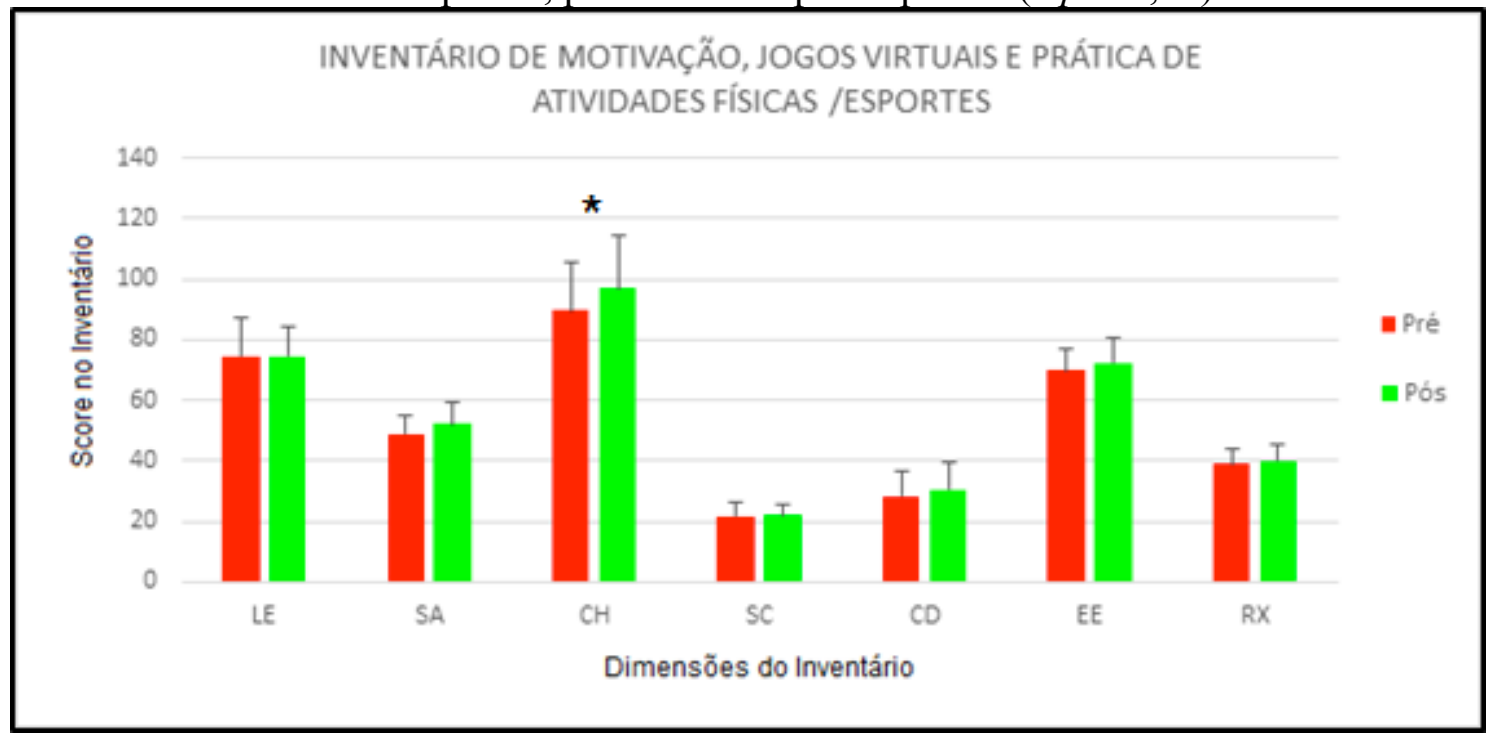

Uma análise mais detalhada dos participantes na Tabela 3 e Figura 2 indica que houve diferença significativa entre as respostas pré e pós para a dimensão Competências e Habilidades $(\mathrm{CH})$, para os participantes Sedentários $(p=0,0039)$ e Fisicamente Ativos ( $p=0,0433)$ e para a dimensão Saúde (SA) para os participantes Fisicamente Ativos ( $p$ $=0,0433)$.

Tabela 3: Teste de variância de Friedman nas condições pré e pós intervenção com EXGs, para os participantes Sedentários, Moderados e Fisicamente Ativos, considerando as sete dimensões do Inventário de Motivação, Jogos Virtuais e Prática de Atividades Físicas/Esportes, $(* p<0,05)$.

Friedman $(p)$

\begin{tabular}{llll}
\hline & Sedentários & Moderados & Fisicamente Ativos \\
\hline Lazer e Entretenimento (LE) & $\mathrm{ns}$ & 0,7728 & $\mathrm{~ns}$ \\
\hline Saúde (SA) & 0,7728 & 0,3865 & $0,0433^{*}$ \\
\hline Competências e Habilidades (CH) & $0,0039^{*}$ & 0,0833 & $0,0433^{*}$ \\
\hline Sociabilidade (SC) & 0,5637 & 0,3865 & 0,3865 \\
\hline Competitividade e Desafios (CD) & 0,1489 & 0,5637 & 0,1489 \\
\hline Estados Emocionais (EE) & 0,7728 & 0,5637 & $\mathrm{~ns}$ \\
\hline Relaxamento (RX) & 0,7728 & $\mathrm{~ns}$ & $\mathrm{~ns}$ \\
\hline
\end{tabular}


Fernanda Carolina Pereira, O Efeito dos Exergames na Motivação para a Prática de Atividade Física... Adriane Beatriz de S. Serapião e

José Silvio Govone

Figura 2: Gráfico representativo dos escores pré e pós em cada dimensão do Inventário de Motivação, Jogos Virtuais e Prática de Atividades Físicas/Esportes, para os participantes Sedentários, Moderados e Fisicamente Ativos, para cada dia de intervenção com EXGs $(* p<0,05)$.

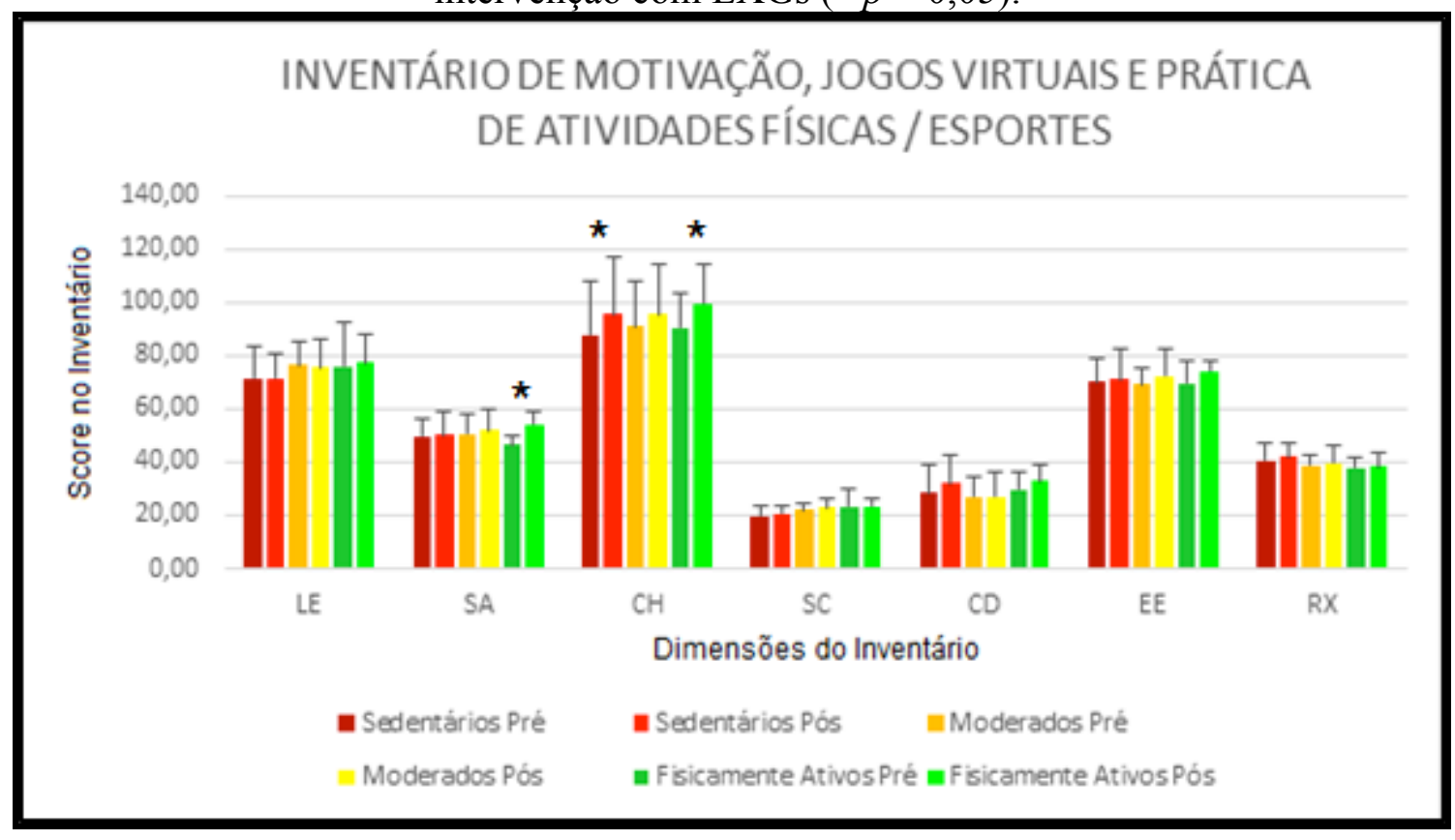

A Tabela 4 e as Figuras 3 e 4 mostram os resultados dos escores pré e pós intervenção com EXGs, em cada dimensão do Inventário de Motivação, Jogos Virtuais e Prática de Atividades Físicas/Esportes, para os participantes Sedentários, Moderados e Fisicamente Ativos, separados nas seções Videogame e Atividades Físicas/Esportes. Nota-se que houve diferença significativa entre as respostas pré e pós para a dimensão Competências e Habilidades $(\mathrm{CH})$, para os participantes Sedentários $(p=0,0143)$ e Moderados $(p=0,0143)$, especificamente na seção Atividades Físicas/Esportes, e para a dimensão Competitividade e Desafios (CD) para os participantes Sedentários $(p=$ 0,0412). 
Fernanda Carolina Pereira, O Efeito dos Exergames na Motivação para a Prática de Atividade Física... Adriane Beatriz de S. Serapião e

José Silvio Govone

Tabela 4: Teste de variância de Friedman para os participantes Sedentários, Moderados e Fisicamente Ativos, considerando as sete dimensões do Inventário de Motivação, Jogos Virtuais e Prática de Atividades Físicas/Esportes, seção Videogame e seção Atividades Físicas/Esportes, $(* p<0,05)$.

\begin{tabular}{l|ll|ll|ll}
\hline Friedman $(p)$ & Sedentários & Moderados & \multicolumn{2}{l}{ Fisicamente Ativos } \\
\hline & Videogame & $\begin{array}{l}\text { Atividades } \\
\text { Físicas/ } \\
\text { Esportes }\end{array}$ & Videogame & $\begin{array}{l}\text { Atividades } \\
\text { Físicas/ } \\
\text { Esportes }\end{array}$ & Videogame & $\begin{array}{l}\text { Atividades } \\
\text { Físicas/ } \\
\text { Esportes }\end{array}$ \\
\hline $\begin{array}{l}\text { Lazer e } \\
\begin{array}{l}\text { Entretenimento } \\
\text { (LE) }\end{array}\end{array}$ & 0,6831 & 0,6831 & 0,6831 & ns & 0,4142 & 0,4142 \\
\hline Saúde (SA) & 0,4142 & 0,2207 & 0,6831 & 0,4142 & 0,1025 & 0,2207 \\
\hline $\begin{array}{l}\text { Competências e } \\
\text { Habilidades (CH) }\end{array}$ & 0,1025 & $0,0143^{*}$ & $n s$ & $0,0143 *$ & 0,1025 & 0,2207 \\
\hline Sociabilidade (SC) & 0,2207 & 0,6831 & 0,6831 & 0,4142 & 0,4142 & 0,6831 \\
\hline $\begin{array}{l}\text { Competitividade e } \\
\text { Desafios (CD) }\end{array}$ & ns & $0,0412^{*}$ & 0,6831 & 0,6831 & 0,4142 & 0,2207 \\
\hline $\begin{array}{l}\text { Estados } \\
\text { Emocionais (EE) }\end{array}$ & 0,6831 & $\mathrm{~ns}$ & 0,6831 & 0,6831 & 0,6831 & 0,6831 \\
\hline Relaxamento (RX) & 0,4142 & 0,6831 & 0,2207 & 0,2207 & 0,4142 & 0,4142 \\
\hline
\end{tabular}

Figura: 3: Gráfico representativo dos escores pré e pós intervenção com EXGs, em cada dimensão do Inventário de Motivação, Jogos Virtuais e Prática de Atividades Físicas/Esportes, para os participantes Sedentários, Moderados e Fisicamente Ativos, na seção Videogame $(* p<0,05)$.

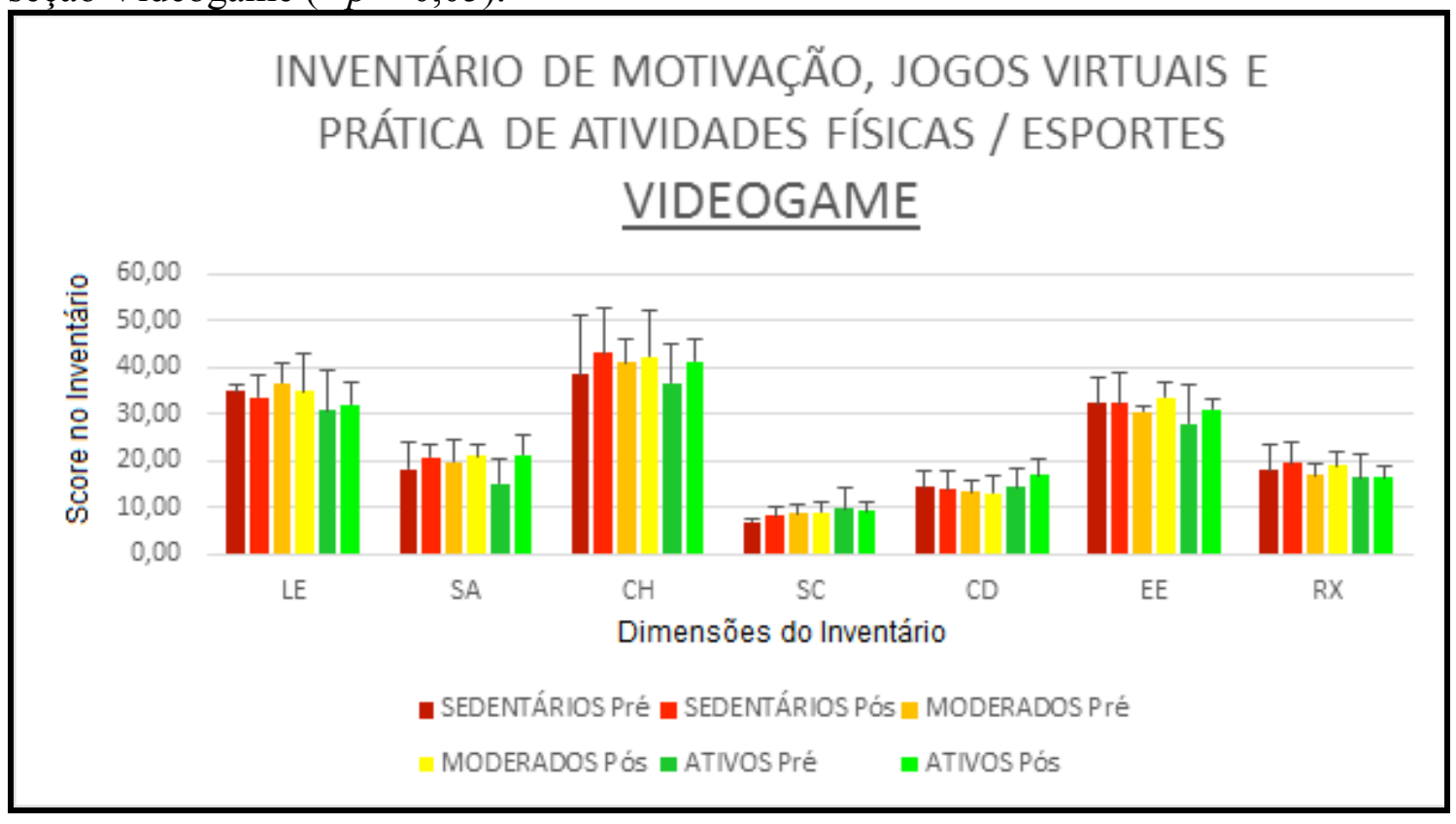


Fernanda Carolina Pereira, O Efeito dos Exergames na Motivação para a Prática de Atividade Física... Adriane Beatriz de S. Serapião e

José Silvio Govone

Figura 4: Gráfico representativo dos escores pré e pós intervenção com EXGs, em cada dimensão do Inventário de Motivação, Jogos Virtuais e Prática de Atividades Físicas/Esportes, para os participantes Sedentários, Moderados e Fisicamente Ativos, na seção Atividades Físicas/Esportes $(* p<0,05)$.

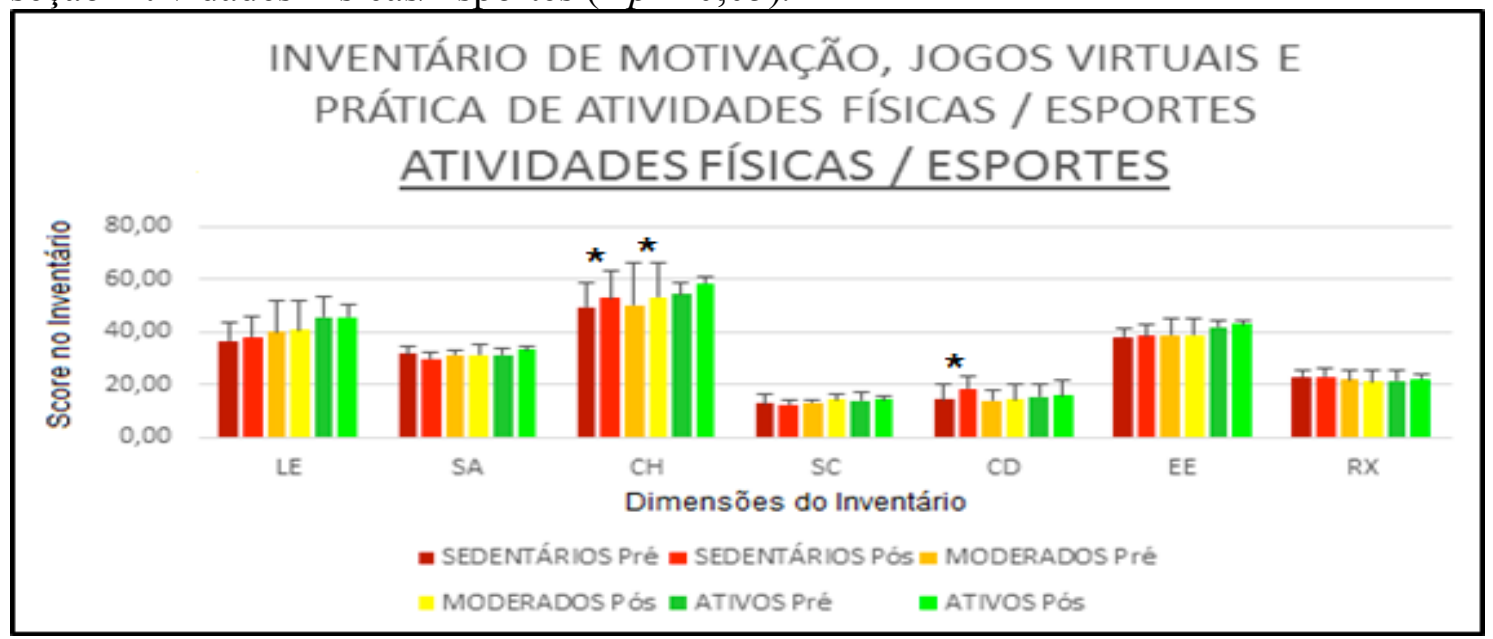

Uma análise mais detalhada dos participantes na Tabela 5 e Figura 5 indica que houve diferença significativa entre as respostas pré e pós para a dimensão Competências e Habilidades $(\mathrm{CH})$, para os participantes Jogadores de Videogame $(p=0,0184)$ e Não Jogadores de Videogame $(p=0,0022)$, indicando que a intervenção com EXGs alterou significativamente as opiniões dos participantes com relação ao tema Competências e Habilidades $(\mathrm{CH})$, independente se aqueles possuem prática ou não com jogos virtuais.

Tabela 5: Teste de variância de Friedman das condições pré e pós intervenção com EXGs, para os participantes Jogadores de Videogame e Não Jogadores de Videogame, considerando as sete dimensões do Inventário de Motivação, Jogos Virtuais e Prática de Atividades Físicas / Esportes, seção Videogame e seção Atividades Físicas / Esportes, $(* p<0,05)$.

\begin{tabular}{lll}
\hline Friedman (p) & & \\
\hline & Jogadores & Não Jogadores \\
\hline Lazer e Entretenimento (LE) & 0,1573 & 0,2386 \\
\hline Saúde (SA) & 0,3458 & 0,8137 \\
\hline Competências e Habilidades (CH) & $0,0184^{*}$ & $0,0022^{*}$ \\
\hline Sociabilidade (SC) & 0,6374 & 0,3458 \\
\hline Competitividade e Desafios (CD) & 0,3458 & 0,3458 \\
\hline Estados Emocionais (EE) & 0,4795 & 0,1573 \\
\hline Relaxamento (RX) & $\mathrm{ns}$ & 0,8137 \\
\hline
\end{tabular}


Fernanda Carolina Pereira, O Efeito dos Exergames na Motivação para a Prática de Atividade Física... Adriane Beatriz de S. Serapião e

José Silvio Govone

Figura 5: Gráfico representativo dos escores pré e pós intervenção com EXGs, em cada dimensão do Inventário de Motivação, Jogos Virtuais e Prática de Atividades Físicas / Esportes, para os participantes Jogadores de Videogame e Não Jogadores de Videogame $(* p<0,05)$.

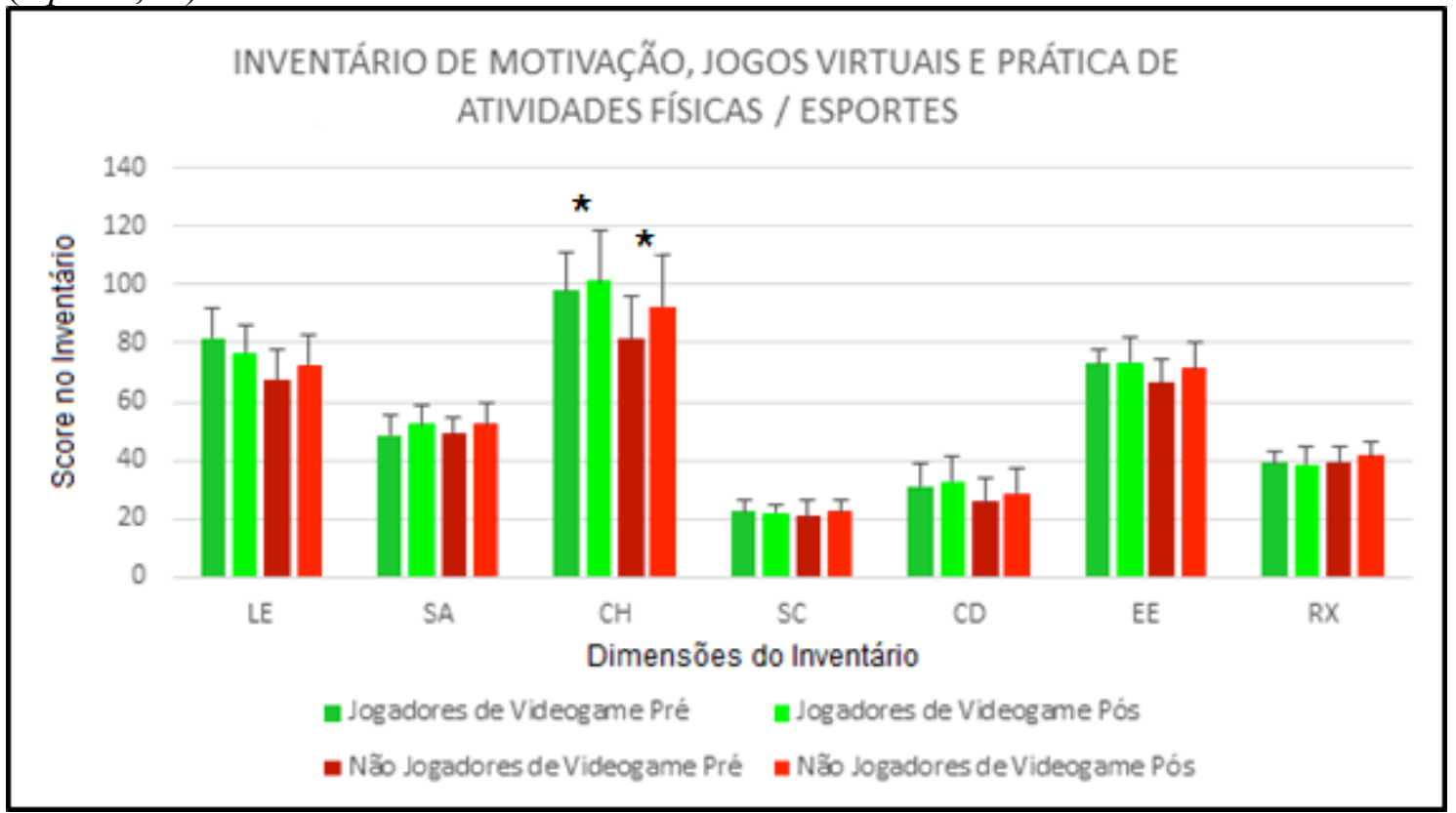

Analisando mais cuidadosamente os participantes na Tabela 6 e Figuras 6 e 7 indica que houve diferença significativa entre as respostas pré e pós para a dimensão Competências e Habilidades $(\mathrm{CH})$, para os participantes Jogadores de Videogame $(p=$ 0,0196) e Não Jogadores de Videogame $(p=0,0077)$, especificamente na seção Atividades Físicas/Esportes, indicando que a intervenção com EXGs alterou significativamente as opiniões dos participantes com relação ao tema Competências e Habilidades $(\mathrm{CH})$ especificamente nas questões voltadas à temática de atividades físicas e esportes, independente se os participantes possuem prática ou não com jogos virtuais. 
Fernanda Carolina Pereira, O Efeito dos Exergames na Motivação para a Prática de Atividade Física... Adriane Beatriz de S. Serapião e

José Silvio Govone

Tabela 6: Teste de variância de Friedman para os participantes Sedentários, Moderados e Fisicamente Ativos, considerando as sete dimensões do Inventário de Motivação, Jogos Virtuais e Prática de Atividades Físicas/Esportes, seção Videogame e seção Atividades Físicas/Esportes, seção Videogame e Seção Atividades Físicas/Esportes, ( ${ }^{*} p$ $<0,05)$.

\begin{tabular}{l|ll|ll}
\hline Friedman (p) & \multicolumn{5}{l}{} \\
\hline & Jogadores de Videogame & $\begin{array}{l}\text { Não Jogadores de } \\
\text { Videogame }\end{array}$ \\
\hline & Videogame & $\begin{array}{l}\text { Atividades } \\
\text { Físicas/ } \\
\text { Esportes }\end{array}$ & Videogame & $\begin{array}{l}\text { Atividades } \\
\text { Físicas/ } \\
\text { Esportes }\end{array}$ \\
\hline Lazer e Entretenimento (LE) & 0,5050 & 0,1824 & 0,5050 & 0,3173 \\
\hline Saúde (SA) & 0,1824 & $\mathrm{~ns}$ & 0,7389 & 0,5050 \\
\hline Competências e Habilidades (CH) & 0,3173 & $0,0196^{*}$ & 0,0956 & $0,0077^{*}$ \\
\hline Sociabilidade (SC) & 0,5050 & $\mathrm{~ns}$ & 0,1824 & $\mathrm{~ns}$ \\
\hline Competitividade e Desafios (CD) & 0,5050 & 0,5050 & 0,7389 & 0,0956 \\
\hline Estados Emocionais (EE) & 0,5050 & 0,7389 & 0,3173 & 0,3173 \\
\hline Relaxamento (RX) & 0,7389 & 0,7389 & 0,5050 & 0,7389 \\
\hline
\end{tabular}

Figura 6: Gráfico representativo dos escores pré e pós intervenção com EXGs, em cada dimensão do Inventário de Motivação, Jogos Virtuais e Prática de Atividades

Físicas/Esportes, para os participantes Jogadores de Videogame e Não Jogadores de Videogame, na seção Videogame $(* p<0,05)$.

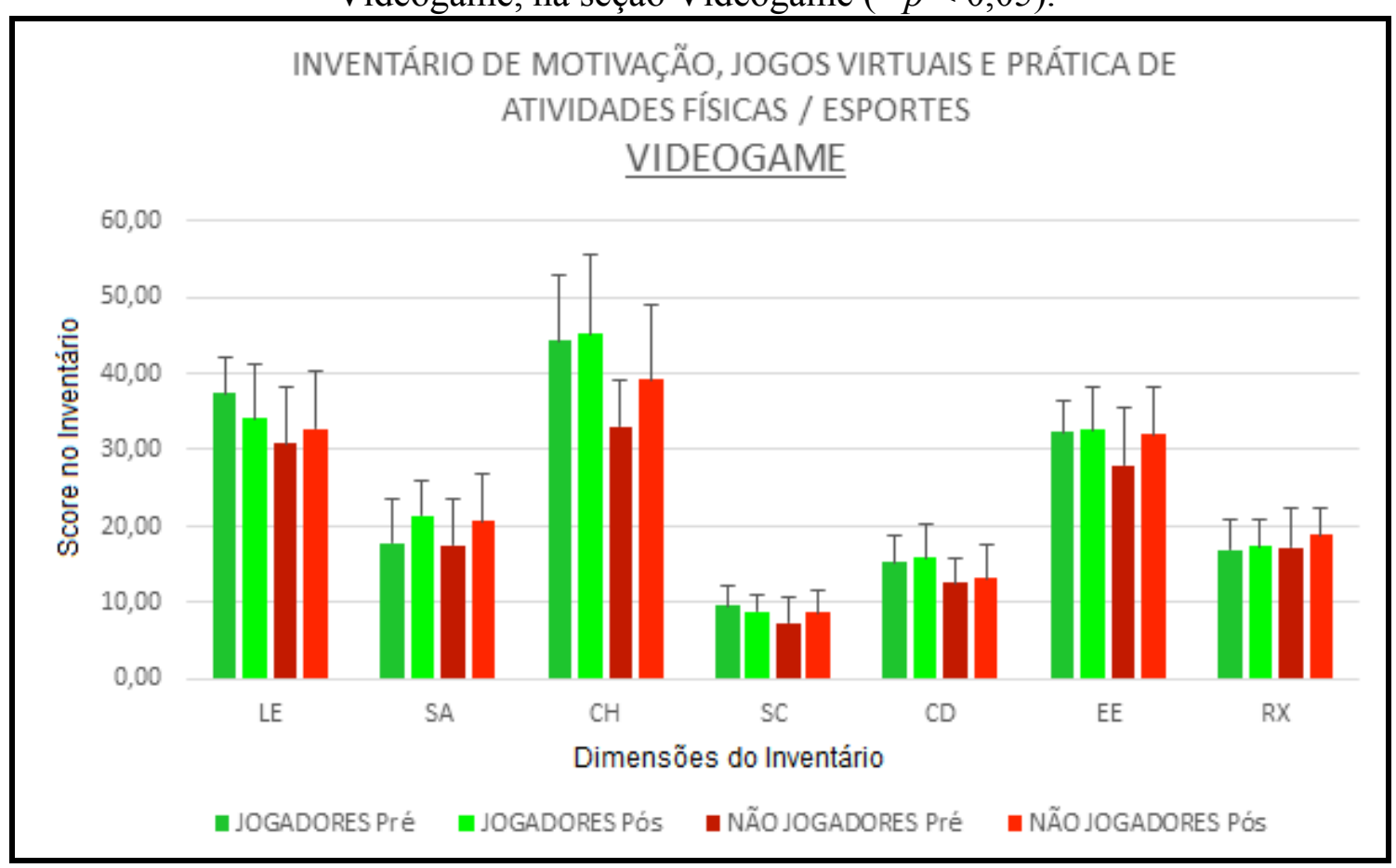


Fernanda Carolina Pereira, O Efeito dos Exergames na Motivação para a Prática de Atividade Física... Adriane Beatriz de S. Serapião e

José Silvio Govone

Figura 7: Gráfico representativo dos escores pré e pós intervenção com EXGs, em cada dimensão do Inventário de Motivação, Jogos Virtuais e Prática de Atividades Físicas/Esportes, para os participantes Jogadores de Videogame e Não Jogadores de Videogame, na seção Atividades Físicas/Esportes $*^{*} p<<$ $0,05)$.

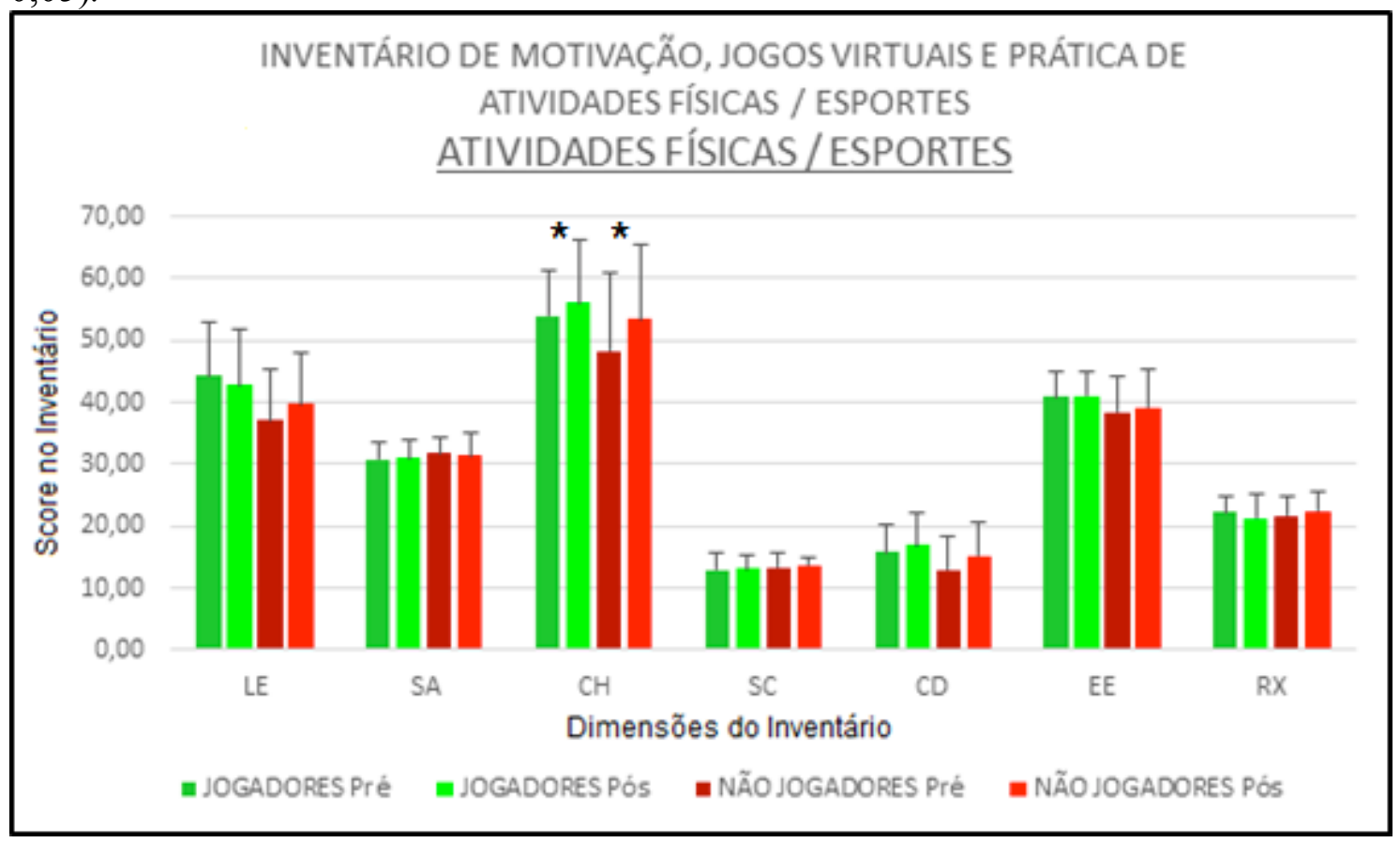

\section{Discussão}

Os EXGs oferecem práticas diferentes, conteúdos diversificados, esportes inabituais, formas divertidas e motivadoras de se abordar os temas nos ambientes educacionais. Além disso, proporcionam às crianças e jovens a oportunidade de experimentar uma maior diversidade de atividades, às quais podem não ter sido expostas de outra forma durante suas vidas. Isto pode contribuir, posteriormente, para um aumento de sua motivação a se envolverem nestas atividades, em clubes desportivos locais ou dentro de suas comunidades (DALEY, 2010).

Os resultados do estudo de Vaghetti et al. (2014), realizado com 64 estudantes de Educação Física, com idades entre 22 e 33 anos, dos cursos de bacharelado e de licenciatura, indicam que a maioria dos estudantes considera que os EXGs podem despertar o interesse por alguma modalidade esportiva e que também podem ser 
Fernanda Carolina Pereira, O Efeito dos Exergames na Motivação para a Prática de Atividade Física... Adriane Beatriz de S. Serapião e

José Silvio Govone

utilizados pelo professor nas diversas faixas etárias nos currículos das escolas e no ensino superior, tanto bacharelado quanto licenciatura.

Epstein et al. (2007) investigaram a interatividade de crianças entre 8 e 12 anos, onde observaram que a prática do jogo Dance Dance Revolution foi mais motivadora do que a execução solitária de movimentos de dança ou o simples ato de assistir televisão.

Analisando os estudos com maior ênfase na prática de atividade física, encontrase que Sun (2013) e Keskinen et al. (2014) verificaram que os EXGs podem contribuir para a realização de atividades físicas, e atuam diretamente na motivação para a prática durante a disciplina de Educação Física em jovens escolares. Além dos estudos de Betker et al. (2006) constatarem que o videogame pode motivar o sujeito à prática de atividade física, Widman, McDonald e Abresch (2006) também encontraram uma maior motivação para prática de atividade física em um grupo de crianças com espinha bífida utilizando um jogo denominado GameCycle.

Benefícios nos estados emocionais e motivação de crianças foram demonstrados em estudos com jogos de dança, como o Dance Dance Revolution (DDR). Ao jogarem DDR, crianças que vivenciam situações de divertimento e aprendizado atingem níveis de atividade física mais elevados no ambiente do jogo DDR, bem como desenvolvem sentimentos de prazer e satisfação (GAO et al., 2012; GAO, PODLOG e HUANG, 2013).

Pode-se notar no presente estudo que os resultados apontaram diferenças significativas para os valores referentes às respostas relacionadas especificamente às atividades físicas e esportes, diferentemente do que aconteceu com os resultados referentes à temática Videogame. Isto sugere que a intervenção com EXGs pode ter influenciado e modificado a ideia que os participantes possuíam sobre as temáticas, se 
Fernanda Carolina Pereira, O Efeito dos Exergames na Motivação para a Prática de Atividade Física... Adriane Beatriz de S. Serapião e

José Silvio Govone

comparados os resultados pré e pós intervenção. Estes resultados proporcionam uma amostra do potencial que os EXGs demonstram possuir e oferecer aos seus usuários, tanto em matéria de entretenimento quanto em proporcionar engajamento em outras atividades da vida cotidiana.

Com o aperfeiçoamento das tecnologias digitais, a realidade virtual passou a ser englobada amplamente por diferentes segmentos tais como entretenimento, saúde, negócios, treinamentos, educação e também atividades físicas. As suas características básicas - a imersão, a interação e o envolvimento - possibilitam pensar em sua utilização como meio de ampliação das formas de ensino-aprendizagem. A realidade virtual nos possibilita conhecer, visitar, aprender sobre lugares distantes ou mesmo aqueles que não existem mais, fruto da evolução da humanidade.

\section{Conclusão}

Os videogames, ícones da cultura digital contemporânea, quando utilizados de forma reflexiva e estratégica, incorporam bons princípios, capazes de dinamizar e diversificar a configuração do ensino-aprendizagem, e adequá-la às exigências da cibercultura. Além disso, para a educação física, os EXGs assumem um significado diferente ao incorporar o movimento corporal, rompendo o paradigma da inatividade dos jogos eletrônicos e criando novas possibilidades de vivências corporais.

O presente estudo proporcionou estabelecer conexões entre a influência das novas tecnologias, com enfoque específico nos jogos virtuais e EXGs, os estados emocionais, mais especificamente a motivação e a prática de exercícios e atividades físicas. 
Fernanda Carolina Pereira, O Efeito dos Exergames na Motivação para a Prática de Atividade Física... Adriane Beatriz de S. Serapião e

José Silvio Govone

\section{REFERÊNCIAS}

BETKER, A. L. et al. Vídeo game-bases exercises for balance rehabilitation: A singlesubject design. Archives of Physical Medicine and Rehabilitation. v. 87, p. 1141$1149,2006$.

CRAIG, C. L. et al. International physical activity questionnaire: 12-country reliability and validity. Medicine and Science in Sports and Exercise, v. 35, n. 8, p. 1381-1395, 2003.

DALEY, A. J. Can exergaming contribute to improving physical activity levels and health outcomes in children? Pediatrics, v. 124, n. 2, p. 763-771, 2010.

ENNIS, C. D. Implications of exergaming for the physical education curriculum in the $21^{\text {st }}$ century. Journal of Sport and Health Science, v. 2, p. 152-157, 2013.

ENNIS, C. D. On their own: preparing students for a lifetime. Journal of Health, Physical Education and Recreation, v. 81, p. 17-22, 2010.

EPSTEIN, L.H. et al. Choice of interactive dance and bicycle games in overweight and non-overweight youth. Annals of Behavioral Medicine, v.33, p. 124-131, 2007.

GAO, Z. et al. Impact of interactive dance games on urban children's physical activity correlates and behavior. Journal of Exercise and Science Fitness, v. 10, p. 107-112, 2012.

GAO, Z.; PODLOG, L.; HUANG, C. Associations among children's situational motivation, physical activity participation, and enjoyment in an active dance video game. Journal of Sport and Health Science, v. 2, p. 122-128, 2013.

KESKINEN, T. et al. Schoolchildren's user experiences on a physical exercise game utilizing lighting and audio. Entertainment Computing. In press, 2014.

MADDISON, R. et al. Feasibility, design and conduct of a pragmatic randomized controlled trial to reduce overweight and obesity in children: The electronic games to aid motivation to exercise (eGAME) study. BMC Public Health, n. 146, p. 1-9, 2009.

SUN, H. Impact of exergames on physical activity and motivation in elementary school students: a follow up study. Journal of Sport and Health Science, v. 2, p. 138-145, 2013.

TROUT, J.; CHRISTIE, B. J. Interactive video games in physical education: rather than contribute to a sedentary lifestyle, these games demand activity from the players. Journal of Physical Education, Recreation and Dance, London, v. 5, n. 78, p. 29-35, 2007.

VAGHETTI, C. A. O. et al. Exergames na Educação Física: ferramentas para o ensino e promoção da saúde. Proceedings of SBGames, XIII SBGames, Porto Alegre, 2014. 
Fernanda Carolina Pereira, O Efeito dos Exergames na Motivação para a Prática de Atividade Física... Adriane Beatriz de S. Serapião e

José Silvio Govone

WANG, X.; PERRY, A. C. Metabolic and Physiologic Responses to Video Game Play in 7- to 10-Year-Old Boys. Archives of Pediatrics and Adolescent Medicine, v. 160, p. 411-415, 2006.

WIDMAN, L. M.; McDONALD, C. M.; ABRESCH, R. T. Effectiveness of an Upper Extremity Exercise Device Integrated With Computer Gaming for Aerobic Training in Adolescents With Spinal Cord Dysfunction. Journal of Spinal Cord Medicine, v. 29, n. 4, p. 363-370, 2006.

\section{Endereço dos Autores:}

Fernanda Carolina Pereira

Universidade Estadual Paulista (UNESP)

Departamento de Estatística, Matemática Aplicada e Computação Avenida 25-A, 1515. Bela Vista

Rio Claro - SP - 13.506-900

Endereço Eletrônico: fernandamarcantonio@gmail.com

Adriane Beatriz de Souza Serapião Universidade Estadual Paulista (UNESP)

Departamento de Estatística, Matemática Aplicada e Computação Avenida 25-A, 1515. Bela Vista

Rio Claro - SP - 13.506-900

Endereço Eletrônico: adriane@rc.unesp.br

José Silvio Govone

Universidade Estadual Paulista (UNESP)

Departamento de Estatística, Matemática Aplicada e Computação Avenida 25-A, 1515. Bela Vista

Rio Claro - SP - 13.506-900

Endereço Eletrônico: jsgovone@gmail.com 